\section{Albero, B., Simonian, S. et Eneau, S. (2019). Des humains \& des machines Hommage aux travaux d'une exploratrice. : Dijon, France : Editions Raison et Passions}

doi:10.18162/fp.2019.a180

\title{
RECENSION
}

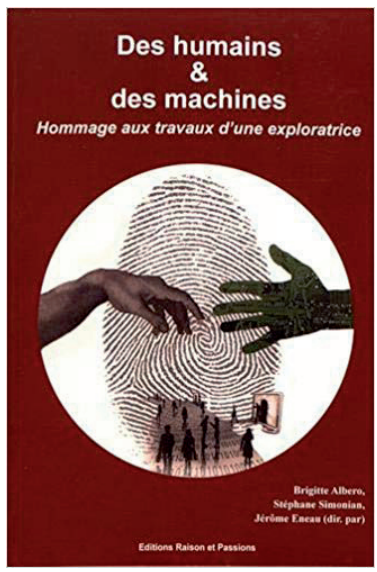

Le titre du nouvel ouvrage dirigé par B.Albero, S. Simonian et J. Eneau suscite d'emblée la curiosité : de quelle exploratrice s'agit-il? Un indice pour nous mettre sur la voie : ce titre fait écho à un ouvrage intitulé Des machines et des hommes : apprendre avec les nouvelles technologies, publié en 1989 par... Monique Linard. "Monique qui??, se demandera-t-on peut-être au Québec. Commençons donc par présenter en quelques points cette grande chercheure française au parcours riche et varié, telle qu'elle apparaît à travers l'ouvrage. Une brève notice biographique à la page 467 nous apprend que Monique Linard a obtenu son doctorat en sciences de l'éducation en 1973, puis qu'elle est devenue professeure à l'Université Paris 10 - Nanterre à partir de 1985. De là, elle amorce une carrière scientifique remarquable qui l'amène à être tour à tour directrice de programme, directrice de département, directrice de centre de recherche et finalement professeure émérite. Mais c'est dès 1962, en tant qu'enseignante d'anglais, que sa curiosité vive et son esprit critique aiguisé l'amènent à s'interroger sur les technologies en éducation sur la base de ses pratiques pédagogiques. La liste de ses publications (p. 468-471 de l'ouvrage) témoigne d'une chercheure prolifique, dont les travaux ont porté sur quatre vagues successives de technologies, depuis l'audiovisuel jusqu'au numérique, en les abordant de manière résolument pluridisciplinaire. En effet, comme le souligne B. Albero en introduction :

Ses thèmes de recherche débordent donc régulièrement les sciences de l'éducation. Elle n'hésite pas à franchir les frontières disciplinaires avec rigueur et prudence pour documenter ses interrogations, étayer ses intuitions, consolider ses analyses : que 
ce soit en linguistique, sémiotique, psychologie, informatique, mais aussi en biologie et neurobiologie, elle enrichit constamment ses références théoriques pluridisciplinaires appuyées sur une littérature internationale. (p. 16)

Pour preuve, l'ouvrage contient trois textes inédits de Monique Linard (p. 473 et suivantes) qui illustrent bien la diversité, la créativité et l'humanisme inlassables qui ont marqué ses travaux. Toutefois, loin d'un ensemble éclaté et confus, son parcours scientifique s'articule autour de principes fédérateurs et structurants, dont il tire une forte cohérence; notamment, une vision large et intégrative de l'apprentissage (en tant qu'acte biologique, psychologique et social) et de la technique (en tant que médiation fonctionnelle, sociale et symbolique de l'activité humaine). C'est certainement cette vision large et fédératrice de la relation entre la société, l'humain, l'apprentissage et la technique qui a permis à Monique Linard d'en rendre compte de manière si productive et originale.

Ses travaux ont assurément marqué le développement du domaine des technologies en éducation en Europe francophone, en jetant les bases de multiples chantiers de recherche. Se pose alors la question de savoir comment couvrir un parcours aussi riche et diversifié. L'ouvrage relève ce défi de manière très convaincante en réunissant une quarantaine de chapitres et autant de chercheurs issus des diverses disciplines des technologies en éducation, ce qui représente un effort considérable de structuration des connaissances. Pour cette raison, il serait vain de rentrer dans une restitution fine des apports et de la complémentarité (bien réels!) de chaque chapitre à la structure d'ensemble. Sur le plan formel, contentons-nous de préciser que ces chapitres sont regroupés en sept parties, qui sont autant de points d'entrée sur les travaux de Monique Linard : «Partie 1 - Hommage à une exploratrice; Partie 2 - Un positionnement épistémologique entre connaissance et action, sujets et artefacts; Partie 3 - Hélices : un modèle de l'activité humaine; Partie 4 - Entre recherche et intervention : des concepts intermédiaires; Partie 5 - Mythes et limites de la vidéoscopie; Partie 6 - Préoccupations praxéologiques en éducation et en formation; Partie 7 - Perspectives de recherche».

Mais qu'on ne s'y trompe pas : cet ouvrage fait honneur à Monique Linard non pas par une restitution point par point de ses travaux, mais plutôt en offrant une vue solide et exhaustive des nombreuses inspirations et ramifications qu'elle a su susciter durant plusieurs décennies. En ce sens, cet ouvrage est autant un hommage qu'un portrait actuel de la diversité et de la richesse que Monique Linard a largement contribué à impulser dans la recherche sur les technologies en éducation. Il se présente comme une synthèse précieuse des fondements scientifiques pluridisciplinaires des technologies en éducation, en abordant à la fois des questions de fond (par exemple, rapport entre technique et société/ humains/apprentissage, notamment dans les parties 2 et 3), des concepts structurants du domaine (par exemple, ceux de dispositif, de médiation et de médiatisation, ou encore d'ingénierie, d'agentivité et d'activité, notamment dans la partie 4), et des modalités d'intervention éducative (voir notamment les parties 5 et 6). Les chapitres relativement courts condensent efficacement les questionnements et les avancées des points abordés en mobilisant les références essentielles de la littérature scientifique. Ainsi, cet ouvrage est largement à la hauteur des trois ambitions que B. Albero annonce en introduction :

1) la production d'un état des lieux des apports de ce champ de recherche mettant en valeur les connaissances acquises pour repérer les travaux prioritaires à conduire à partir de ce socle et les enseigner dans les cursus universitaires de formation à des fins de transmission; 2) l'identification de débats à conduire sur les paradigmes émergents associés au numérique, que ce soit en termes de positionnement 
épistémologique, de cadres théoriques, de méthodes, de terrains et de pratiques; 3) la formalisation d'une réflexion sur la contribution des disciplines représentées dans cet ouvrage à la production de ressources et d'environnements de formation, selon une finalité de développement. (p. 15)

Preuve de cette volonté de contribuer à la structuration des connaissances, un glossaire en fin d'ouvrage (p.581-597) reprend de manière synthétique mais fournie les principaux concepts qui traversent l'ouvrage.

Au final, cet ouvrage promet de devenir une référence structurante de la recherche sur les technologies en éducation, et s'offre du même coup comme une très belle introduction pour les jeunes chercheurs et chercheures du domaine (d'Europe francophone et d'ailleurs!). À une époque où la qualité scientifique est de plus en plus réduite à des indicateurs chiffrés de performance, Monique Linard devrait être très fière car, au-delà de toutes les subventions possibles, au-delà de la course à la publication et au-delà de la visibilité médiatique et politique, que subsiste-t-il d'une grande chercheure, si ce n'est son pouvoir d'inspiration d'un si bel ouvrage en son hommage?

\section{Pour citer cet article}

Collin, S. (2019). Albero, B., Simonian, S. et Eneau, S. (2019). Des humains \& des machines Hommage aux travaux d'une exploratrice. : Dijon, France : Editions Raison et Passions Formation et profession. 27(3), 159-161.

http://dx.doi.org/10.18162/fp.2019.a180 